\title{
Mecanismo Eficiente para Disseminação do Tráfego de Controle em Redes de Sensores Sem Fio Cluster-Tree baseadas no IEEE 802.15.4
}

\author{
Miguel Lino*, Vitor Vasconcelos*, Erico Leão*, André Soares*, \\ Carlos Montez ${ }^{\dagger}$, Ricardo Moraes ${ }^{\dagger}$ e Francisco Vasques ${ }^{\ddagger}$ \\ * Departamento de Computação, Universidade Federal do Piauí, Brasil \\ Email: \{miguelneto0019,vitorvmvbr\}@gmail.com; \{ericoleao,andre.soares\}@ufpi.edu.br \\ $\dagger$ Universidade Federal de Santa Catarina, Brasil \\ Email: \{carlos.montez,ricardo.moraes\}@ufsc.br \\ $\ddagger$ INEGI, Faculdade de Engenharia, Universidade do Porto, Portugal \\ Email: vasques@fe.up.pt
}

\begin{abstract}
Resumo-As normas IEEE 802.15.4 e ZigBee definem a pilha de protocolos amplamente utilizada na operação de Redes de Sensores sem Fio (RSSF). A topologia cluster-tree, suportada por estas normas, oferece recursos como escalabilidade, sendo apontada pela literatura como a mais adequada para a implementação de aplicações de RSSFs em larga escala. Entretanto, essas redes podem sofrer com congestionamentos e atrasos devido aos seus diferentes padrões de tráfego. Por um lado, são usualmente priorizadas as mensagens do tráfego de monitoramento geradas por nós sensores, que seguem em direção ao coordenador da rede (tráfego upstream); por outro, sofrem maiores atrasos as mensagens de controle que seguem o tráfego inverso (tráfego downstream). Este artigo propõe um mecanismo eficiente para a rápida disseminação do tráfego de controle em RSSFs clustertree. A ideia principal consiste em combinar um esquema de escalonamento híbrido periódico, com a definição de janelas de oportunidade Top-Down, com um esquema de configuração adequada dos parâmetros CSMA-CA (Carrier Sense Multiple Access with Collision Avoidance) para os nós coordenadores, a fim de possibilitar uma rápida disseminação das mensagens de controle, sem gerar impactos relevantes para o típico tráfego de monitoramento. Resultados de simulações mostram que a definição de janelas de oportunidades Top-Down, aliada com uma adequada configuração de valores $\operatorname{macMinBE}$ e $\operatorname{macMax} B E$ para nós coordenadores, podem reduzir significativamente os atrasos de comunicação e aumentar a taxa de entrega de mensagens de controle, sem interferir de forma relevante nas métricas do tráfego de monitoramento, mesmo em cenários de grande densidade de nós sensores.
\end{abstract}

\section{INTRODUÇÃO}

As Redes de Sensores sem Fio (RSSFs) são redes ad hoc especiais que possuem como peculiaridades dispositivos de baixa potência, baixo custo e que operam em baixas taxas de transmissão, sendo capazes de detectar, processar e enviar informações de variáveis relacionadas ao ambiente nos quais são implantadas [1]. Essas características tornam esse tipo de rede uma opção atraente para uma variedade de aplicações em larga escala, tais como: monitoramento ambiental e industrial, agricultura de precisão e sistemas veiculares [2]-[4].

O conjunto de normas IEEE 802.15.4 [5] e ZigBee [6] suportam um tipo especial de topologia de rede peer-to- peer conhecida como cluster-tree (árvore de agrupamentos), que oferece recursos, tais como: baixo consumo de energia, escalabilidade e comunicação em tempo real. Tais redes são apontadas como uma das topologias mais adequadas para implementar RSSFs de larga escala [7], [8]. Além de permitir a sincronização da comunicação entre os dispositivos, a topologia cluster-tree implementa formações de redes complexas, onde os nós sensores são organizados em agrupamentos vizinhos (clusters), coordenados por um único nó chamado de coordenador de cluster (Cluster-Head - $\mathrm{CH}$ ). Os clusters são interconectados através de seus $\mathrm{CHs}$, formando assim uma estrutura de rede hierárquica multicluster.

Apesar de uma série de vantagens oferecidas pela topologia cluster-tree, tais como operação com eficiência energética e facilidade de implementação de mecanismos de agregação ou fusão de dados, essas redes podem sofrer graves problemas de atrasos de mensagens e congestionamentos, devido ao padrão de convergência dos tráfegos de comunicação. Tal comportamento, por um lado, prioriza a disseminação de informações geradas por nós sensores em níveis inferiores de uma topologia em árvore, que convergem (tráfego upstream) em direção ao nó principal coordenador da rede, chamado Coordenador PAN (Personal Area Network). Por outro lado, mensagens de controle seguem o caminho inverso (tráfego downstream), podendo sofrer assim maiores atrasos. Aplicações de larga escala frequentemente necessitam de manutenção ou atuação periódica, o que implica no envio de mensagens de controle a partir de nós coordenadores para os demais nós da rede [9].

Dentro deste contexto, este artigo propõe um mecanismo de comunicação eficiente para a rápida disseminação de mensagens de controle (tráfego downstream) em RSSFs cluster-tree baseadas nas normas IEEE 802.15.4 e ZigBee, que combina dois esquemas principais: 1) um esquema de escalonamento híbrido, que periodicamente cria janelas de oportunidade de comunicação para o tráfego de controle durante um intervalo mínimo de tempo; e 2) um esquema de configuração eficiente de parâmetros CSMA-CA para nós coordenadores, a fim de 
priorizar o acesso ao meio de cluster-heads, resultando numa maior probabilidade de transmissões de mensagens de controle durante o período da janela de oportunidade.

O restante deste artigo está organizado como segue: Seção II apresenta as principais características de redes cluster-tree e o mecanismo de acesso ao meio CSMA-CA. Seção III sumariza os trabalhos mais relevantes para o mecanismo proposto neste artigo. Na Seção IV, o mecanismo proposto para rápida disseminação do tráfego de controle é apresentado, detalhando seus principais esquemas. A Seção V apresenta os resultados obtidos através de avaliação por simulação. Finalmente, Seção VI apresenta as conclusões e trabalhos futuros.

\section{Redes De SEnsores SEM Fio Cluster-Tree}

\section{A. As Normas IEEE 802.15.4/ZigBee}

Uma rede cluster-tree é construída através de uma árvore de múltiplos clusters que podem implementar ciclos de trabalho reduzidos, permitindo uma economia de energia dos seus nós associados. Basicamente, uma rede cluster-tree é formada por cluster-heads (CHs), responsáveis por coordenar cada cluster e promover a sincronização entre seu grupo de nós filhos, através de quadros de sinalização (beacons). Os $\mathrm{CHs}$ podem ser conectados entre si a fim de formar uma estrutura hierárquica de múltiplos clusters interligados.

As redes cluster-tree operam no modo de comunicação com beacons. No modo com beacons, a comunicação dentro de um cluster é organizada com base numa estrutura chamada de superframe. Um superframe é limitado por quadros beacons que são periodicamente transmitidos por cluster-heads. Esses beacons sincronizam os nós de cada cluster, identificam o PAN e delimitam a estrutura do superframe. A Figura 1 ilustra a estrutura do superframe.

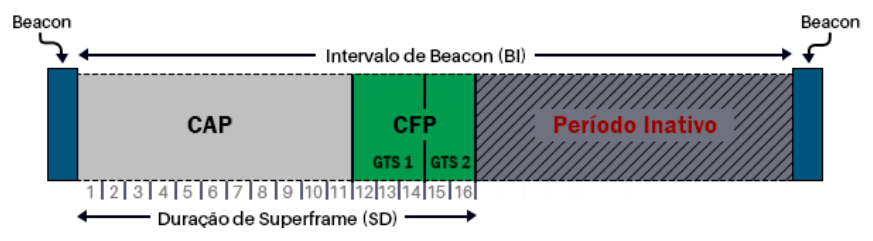

Figura 1. A estrutura do superframe.

Basicamente, um superframe é composto por duas partes: parte ativa e a parte inativa. Durante a parte inativa, nós podem entrar em modo de baixo consumo a fim de economizar energia. A parte ativa é subdividida em dois períodos: Período de Acesso com Contenção (Contention Access Period - CAP) e o Período Livre de Contenção (Contention-Free Period - CFP). Durante o CAP, os nós que desejam se comunicar, competem com outros dispositivos utilizando o mecanismo de acesso ao meio CSMA-CA. Já no CFP, nós sensores podem solicitar slots GTS (Guaranteed Time Slots) para transmissões sem contenção com baixa latência ou largura de banda garantida.

A estrutura do superframe é definida pelos parâmetros macBeaconOrder (BO) e macSuperframeOrder (SO). Esses parâmetros definem o Intervalo de Beacons (BI) e a Duração do Superframe (SD), respectivamente. BI determina o intervalo no qual um nó coordenador deve transmitir periodicamente seus quadros de beacons. Por sua vez, SD determina o tamanho da porção ativa do superframe. Os valores de BI e SD são definidos através da Equação 1:

$$
\begin{aligned}
& B I=\text { aBase } S D \times 2^{B O}, \\
& S D=\text { aBase } S D \times 2^{S O}, 0 \leq S O \leq B O \leq 14
\end{aligned}
$$

onde aBaseSD (aBaseSuperframeDuration) define a duração mínima de um superframe (por padrão, ele corresponde a 960 símbolos, que é equivalente a 15,36 $\mathrm{ms}$, assumindo uma banda de frequência de $2,4 \mathrm{GHz}$ ).

\section{B. Protocolo CSMA/CA}

RSSFs cluster-tree baseadas nas normas IEEE 802.15.4 e ZigBee, operando no modo de comunicação com beacons, utilizam o algoritmo CSMA-CA (slotted) como mecanismo de controle de acesso ao meio. Durante o período CAP de um cluster (SD), os dispositivos que desejam transmitir dados devem competir entre si para terem acesso ao meio, ou seja, executam individualmente uma série de instruções que possam permitir seu acesso ao meio de forma organizada, a fim de evitar possíveis colisões.

Para programar o acesso ao meio e auxiliar nas tomadas de decisões, o protocolo CSMA-CA define três variáveis:

- Backoff Exponent (BE): variável utilizada no cálculo de um tempo aleatório dentro do intervalo $\left[0,2^{B E}-1\right]$, chamado de backoff, durante o qual o dispositivo deve esperar para checar o estado do canal (CCA - Clear Channel Access).

- Contention Window (CW): número de backoffs no qual o canal deve ser classificado como disponível pelo CCA, antes de se iniciar qualquer transmissão.

- Number of Backoffs (NB): número de tentativas falhas de acesso ao meio feitas pelo algoritmo CSMA-CA. Caso $N B>$ macMaxCSMABackoff, a mensagem é reportada como falha.

De acordo com [10], o comportamento do protocolo CSMACA é afetado por quatro parâmetros durante sua inicialização: minimum backoff exponent (macMinBE), maximun backoff exponent (macMaxBE), valor inicial de $C W$ (CWinit) e maximum backoffs number (macMaxCSMABackoffs).

Dentre estes parâmetros de inicialização, o $\operatorname{macMinBE}$ e o macMaxBE provocam maior influência no desempenho de RSSFs [11], pois definem os valores para $B E$, que determinam o intervalo $\left[0,2^{B E}-1\right]$ de atribuição de uma duração aleatória de períodos de backoffs para os quais um nó deve aguardar antes de tentar acessar o canal de comunicação sem fio.

Dessa forma, nós sensores que utilizam valores de $B E$ mais altos podem usualmente aguardar tempos maiores para acessar o canal sem fio, o que consequentemente pode aumentar a taxa de sucesso de transmissão, por reduzir a probabilidade de dois ou mais nós transmitirem mensagens ao mesmo tempo, gerando assim colisões. Por outro lado, nós com valores de $B E$ menores aguardam um menor tempo de contenção para acessar o canal. É importante destacar que, se vários nós em 
contenção tiverem valores $B E$ pequenos, isso poderá resultar em uma maior quantidade de nós tentando acessar o canal ao mesmo tempo, aumentando assim a probabilidade de colisões.

Neste contexto, é possível obter diferentes níveis de prioridade para nós sensores de acordo com o número de períodos de backoffs que um nó necessita aguardar para acessar o canal sem fio. Por exemplo, um nó que aguarda um único período de backoff possui maior prioridade de acesso ao canal em relação a um nó que necessita aguardar cinco períodos de backoffs.

\section{TRABALHOS RELACIONADOS}

Vários trabalhos têm apresentados diferentes abordagens dentro do contexto de melhoria do desempenho de fluxos de mensagens para RSSFs. As propostas abordam várias questões, tais como: modelos analíticos, esquemas de escalonamento de períodos ativos e configuração de parâmetros do algoritmo CSMA-CA em redes cluster-tree de larga escala.

Em [12], os autores propõem o algoritmo Superframe Duration Scheduling (SDS), que é apresentado dentro da abordagem Time Division Beacon Frame Scheduling (TDBS). A ideia básica do TDBS é escalonar quadros de beacons durante os períodos inativos de clusters vizinhos, a fim de evitar colisões de beacons entre clusters. Dessa forma, os clusters são ordenados sequencialmente com base em suas durações de superframes dentro de um ciclo principal periódico. No entanto, essa abordagem não especifica nenhum mecanismo para priorizar um determinado tipo de padrão de tráfego.

Por outro lado, os trabalhos propostos em [13] e [14] fornecem abordagens para priorizar um conjunto específico de fluxos de mensagens. Em [13], os autores apresentam a abordagem Time-Division Cluster Scheduling (TDCS), que além de evitar colisões entre clusters, também atende requisitos de comunicação fim-a-fim para fluxos de mensagens limitados por tempo. A abordagem TDCS define um escalonamento viável que considera as restrições temporais e de recursos de um conjunto de tarefas.

Por sua vez, [14] apresenta a abordagem Dynamic Cluster Scheduling (DCS) para modificar dinamicamente o escalonamento e fornecer Qualidade de Serviço (QoS) para um conjunto de fluxos de mensagens. A ideia básica do DCS é reorganizar em tempo de execução o escalonamento de clusters, além de adaptar o tamanho das durações de superframe usando o espaço livre disponível, aumentando a largura de banda dos clusters envolvidos em um fluxo de mensagens específico. Embora essas abordagens priorizem fluxos específicos, elas não fornecem nenhum mecanismo para priorizar mensagens de controle para redes cluster-tree.

Em [9], é formulada a abordagem Low-latency Two-way Beacon Scheduling (LTBS) para redes ZigBee. O LTBS é uma abordagem de escalonamento de beacons, na qual os nós tentam obter vagas para o tráfego upstream e downstream de baixa latência, evitando interferências de outros clusters. No entanto, a estrutura do superframe original baseada na norma IEEE 802.15.4 é modificada para permitir que clusterheads transmitam dois beacons em direções diferentes: um para upstream e outro para a direção downstream. Além disso, embora a latência da rede seja reduzida, ela não considera nenhuma priorização de fluxo de mensagens.

Em relação ao protocolo CSMA-CA, a literatura aponta que é possível melhorar o desempenho de redes IEEE 802.15.4 ao utilizar parâmetros CSMA-CA adequados com as necessidades das aplicações da rede. Por exemplo, em [15], os autores definem classes de serviço para redes IEEE 802.15.4, onde cada tipo/classe de tráfego possui seus próprios atributos/parâmetros definidos de maneira particular. Os autores associaram diferentes valores para $C W$, macMinBE e macMaxBE para filas de maior e menor prioridade.

Neste mesmo ínterim, em [11] e [16], os autores propõem redes que promovem níveis diferenciados de QoS. Em [11], os autores propõem uma otimização no processo de associação dos nós sensores em redes IEEE 802.15.4. Já em [16], os autores propõem um mecanismo de ajuste dos parâmetros CSMACA, a fim de garantir QoS e serviços diferenciados para fluxos de mensagens gerados por dispositivos em aplicações de smart grid. Entretanto, nenhum desses trabalhos endereçam o problema de priorizar diferentes tipos de fluxos de mensagens em redes cluster-tree, principalmente mensagens de controle (tráfego downstream).

\section{MeCAnismo EFICIENTE PARA DisseminaÇÃo DE Tráfego de Controle em Redes Cluster-Tree}

Este trabalho propõe um mecanismo de comunicação eficiente para uma rápida disseminação do tráfego de controle (tráfego downstream) em RSSFs cluster-tree baseadas nas normas IEEE 802.15.4 e ZigBee. O objetivo principal da proposta é garantir QoS para as mensagens de controle geradas pelos nós coordenadores, sem impactar de forma relevante no típico tráfego de monitoramento gerado periodicamente pelos nós sensores.

Basicamente, o mecanismo proposto considera dois esquemas principais: 1) um esquema de escalonamento híbrido periódico de clusters que define janelas de oportunidade TopDown, a fim de favorecer a disseminação do fluxo de mensagens de controle; e 2) um esquema de configuração eficiente dos parâmetros CSMA-CA para os nós coordenadores, a fim de aumentar a probabilidade de acesso ao meio desses dispositivos, promovendo assim uma diminuição do tempo de disseminação e o aumento da taxa de sucesso de entrega de mensagens de controle ao longo da rede cluster-tree.

As próximas subseções descrevem o problema de disseminação do tráfego de controle e os mecanismos propostos.

\section{A. Descrição do Problema}

Este trabalho considera um cenário típico de monitoramento de uma RSSF, onde um conjunto de $n$ nós sensores são implantados de forma aleatória ao longo de um ambiente de larga escala. Os nós sensores são agrupados em clusters, conforme a topologia cluster-tree baseada nas normas IEEE 802.15.4/ZigBee. Cada cluster é coordenado por um único Cluster-Head - $\mathrm{CH}$ (incluindo o coordenador PAN). Cada $\mathrm{CH}$ envia periodicamente quadros de beacons para sincronizar seus 
nós filhos associados e gerenciar as atividades de comunicação dentro de seu cluster.

$\mathrm{O}$ conjunto de $\mathrm{CHs}$ da rede cluster-tree é caracterizado por: $C H_{i}=\left(S D_{i}, B I_{i}\right), 1 \leq i \leq n_{C H}$; onde $S D_{i}$ e $B I_{i}$ correspondem à duração de superframe e ao intervalo de beacons para o cluster-head $\mathrm{CH}_{i}$, respectivamente. Além disso, é assumido que todos os cluster-heads possuem o mesmo $\mathrm{BI}$ e as durações de superframes para os CHs são definidas considerando o esquema de alocação proposto em [17].

Após serem implantados no ambiente, nós sensores são responsáveis por detectar variáveis físicas ao longo da sua área de cobertura e enviar esses dados de monitoramento para um nó especial denominado de nó sink. O nó sink é responsável por receber informações coletadas de nós sensores e tem a função de atuar como interface com usuários finais ou como gateway para outras redes. Os dados de monitoramento podem ser usados para atividades de controle/atuação. Neste trabalho, é assumido que o coordenador PAN atua como nó sink para a rede cluster-tree. A Figura 2 ilustra o ambiente considerado neste trabalho.

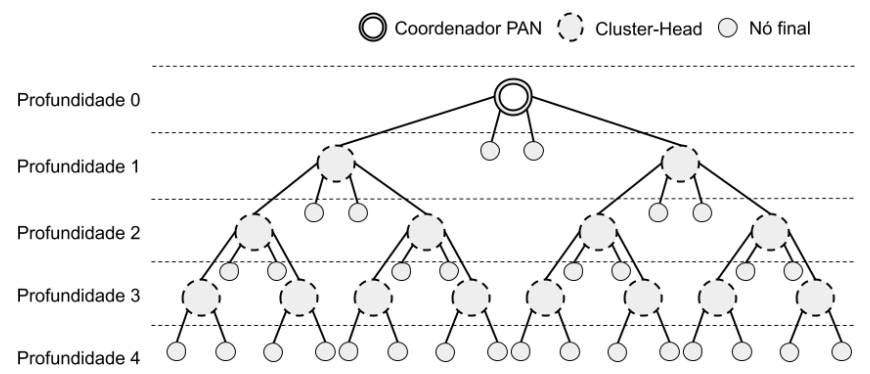

Figura 2. Estrutura de uma rede cluster-tree.

A fim de evitar colisões entre clusters, os períodos ativos dos clusters são previamente escalonados de acordo com um esquema de escalonamento de beacons por divisão de tempo [12]. Dentro desse contexto, o escalonamento de beacons utilizado pode priorizar (ou não) um determinado comportamento de tráfego, tornando-se assim um problema de otimização em redes cluster-tree. Por exemplo, o escalonamento bottom-up prioriza o tráfego de monitoramento (tráfego upstream), pois as durações de superframe são ordenadas a partir dos clusters mais profundos até alcançar o coordenador do PAN (profundidade por profundidade). Ao contrário, o escalonamento top-down prioriza o tráfego de controle (tráfego downstream), pois os SDs de cada cluster são ordenados a partir do coordenador PAN em direção aos clusters mais profundos.

Em redes cluster-tree, todas as comunicações são realizadas com base no roteamento em árvores hierárquicas, ou seja, mensagens irão seguir caminhos definidos pelas relações paifilho dos nós sensores em direção ao coordenador PAN (nó sink). Desta forma, o uso de esquemas de escalonamento bottom-up é satisfatório a fim de priorizar o típico tráfego de monitoramento destas redes. Entretanto, essas redes podem enfretar problemas típicos, tais como: atrasos de mensagens e esgotamento de buffers, que acarretam em congestionamento de rede. Assim, atividades de controle periódicas são frequentemente necessárias para permitir reconfigurações de rede, o que torna o problema de priorização de mensagens de controle de extrema relevância.

\section{B. Esquema de Escalonamento Híbrido para Redes Cluster- tree}

Considerando o problema descrito, este trabalho propõe um esquema de escalonamento híbrido baseado na abordagem proposta em [18], que alterna periodicamente o modelo de escalonamento de redes cluster-tree. Diferentemente de [18], que propõe a disseminação de poucas mensagens de controle via broadcast, o objetivo desse esquema é criar Janelas de oportunidade Top-Down (JTD) periódicas, com tamanho $J T D_{\text {tam }}$ e periodicidade $J T D_{\text {periodic }}$ bem definidos, a fim de permitir a disseminação otimizada de fluxos de tráfego de controle ao longo da rede através de conteção. A Figura 3 ilustra o esquema de escalonamento híbrido proposto.

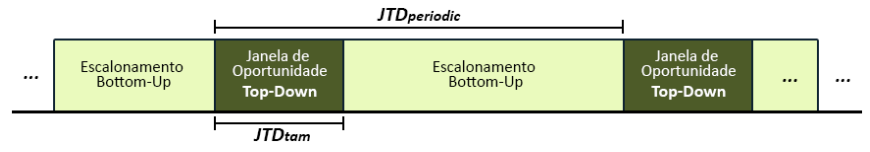

Figura 3. Esquema de escalonamento híbrido periódico, com Janelas de Oportunidade Top-Down para disseminação do tráfego de controle.

Inicialmente, a rede cluster-tree é escalonada seguindo uma abordagem de escalonamento bottom-up. Esse esquema de escalonamento, além de evitar colisões de quadro de beacons entre $\mathrm{CHs}$, tende a priorizar os fluxos de mensagens de monitoramento gerados periodicamente pelos nós sensores em direção ao nó sink da rede (coordenador PAN). Assim, para cada $\mathrm{CH}$, é definido um offset (intervalo de tempo) específico que corresponde ao tempo inicial para a transmissão dos seus quadros de beacons.

No esquema de escalonamento híbrido proposto, $\mathrm{CHs}$ reescalonam a rede a cada intervalo $J T D_{\text {periodic, }}$ através da definição de offsets especiais (Escalon $a_{T D}$ ), alterando a abordagem de escalonamento bottom-up inicial para a abordagem Top-Down, procurando assim priorizar o tráfego de controle. Para reescalonar a rede, cada $\mathrm{CH}$ calcula o offset Escalona ${ }_{T D}$ através da Equação 2:

$$
\text { Escalona }_{T D}=2 \times B I-2 \times \text { off } \operatorname{set}\left[\mathrm{CH}_{i}\right]-S D\left[\mathrm{CH}_{i}\right],
$$

onde $B I$ é o intervalo de beacons, e os valores off set $\left[\mathrm{CH}_{i}\right]$ e $S D\left[C H_{i}\right]$ são o offset inicial e a duração de superframe para o cluster-head $\mathrm{CH}_{i}$, respectivamente.

Dessa forma, aplicando a Equação 2, uma janela de oportunidade com escalonamento Top-Down (JTD) é definida para os clusters, que tende a priorizar o tráfego de controle gerado a partir do coordenador PAN em direção a todos os $\mathrm{CHs}$ da rede. A duração da janela de oportunidade JTD $\left(J T D_{\text {tam }}\right)$ é baseada no comportamento do tráfego de controle e é definida pela Equação 3:

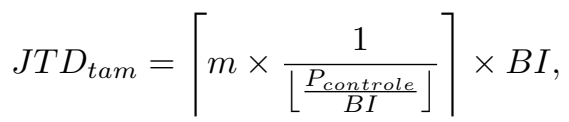


onde $m$ é o número de mensagens de controle a ser enviado a partir do coordenador PAN para todos os $\mathrm{CHs}, P_{\text {controle }}$ é a periodicidade das mensagens de controle, $B I$ é o intervalo de beacons e $\left\lfloor\frac{P_{\text {controle }}}{B I}\right\rfloor$ corresponde ao número máximo de mensagens de controle geradas dentro de um intervalo de beacons. Note que o tamanho da janela de oportunidade ( $J T D_{\text {tam }}$ ) é expressado em números de intervalos de beacons. Após encerrar o período $J T D_{\text {tam }}$ da janela de oportunidade Top-Down, a rede é reescalonada de volta para a abordagem de escalonamento bottom-up inicial, voltando a priorizar o tráfego de monitoramento. Dessa forma, cada $\mathrm{CH}$ calcula seu offset inicial $\left(\right.$ Escalona $\left._{B U}\right)$ através da Equação 4:

$$
\text { Escalona }_{B U}=2 \times \text { offset }[\mathrm{CH} i]+S D[C H i]
$$

É importante salientar que para fazer a alteração do escalonamento da rede (Equações 2 e 4), cada $\mathrm{CH}$ precisa apenas de informações locais. Além disso, a periodicidade da janela de oportunidade ( $\left.J T D_{\text {periodic }}\right)$ é um parâmetro definido de acordo com as necessidades específicas de reconfiguração/atuação impostas pelas aplicações de RSSFs. O esquema de escalonamento híbrido proposto neste trabalho pode ser adequado para aplicações de larga escala que requerem manutenção periódica ou necessitam realizar sistematicamente tarefas de controle, tais como sincronização de clock [19] ou controle de congestionamento [20].

\section{Configuração dos Parâmetros CSMA/CA para $\mathrm{CHs}$}

Ao criar janelas de oportunidade Top-Down periódicas, mensagens de controle podem ser enviadas ao longo da rede cluster-tree e alcançar todos os $\mathrm{CHs}$ dentro de um único intervalo de beacons. Este trabalho assume que as mensagens de controle são propagadas ao longo dos $\mathrm{CHs}$ utilizando o mecanismo de comunicação indireta do padrão IEEE 802.15.4 [5], ou seja, o coordenador anuncia em seu beacon a existência de dados pendentes, que são solicitados e transmitidos ao $\mathrm{CH}$ filho durante o CAP utilizando o protocolo CSMA-CA.

Entretanto, durante a janela de oportunidade Top-Down, o tráfego de monitoramento ainda permanece ativo, o que implica que nós sensores continuam a enviar seus dados dentro dos períodos ativos dos seus clusters (utilizando o protocolo CSMA-CA). Dessa forma, apesar da janela JTD priorizar o tráfego downstream e permitir a propagação de mensagens de controle dentro de um intervalo de beacons, nós coordenadores deverão competir pelo canal sem fio para enviar as mensagens de controle ao longo da rede.

Dessa forma, este trabalho propõe um esquema de configuração eficiente dos parâmetros CSMA-CA para os nós coordenadores durante as janelas de oportunidade Top-Down, aumentando assim a probabilidade desses dispositivos acessarem o canal sem fio. Com isso, este esquema pretende diminuir os atrasos de disseminação de mensagens e aumentar a taxa de sucesso de entrega para o tráfego de controle em redes cluster-tree.

Ao iniciar a janela de oportunidade JTD, nós coordenadores utilizam uma configuração diferenciada para os parâmetros
macMinBE e macMaxBE para o envio específico de mensagens de controle. Quando recebem um beacon com mensagens de controle pendentes, os $\mathrm{CHs}$ filhos solicitam as mensagens de controle pendentes alterando seus valores dos parâmetros macMinBE para 5 e macMaxBE para $8 . \mathrm{O} \mathrm{CH}$ pai ao receber solicitações de dados pendentes, envia as mensagens de controle para seus CHs filhos configurando ambos seus parâmetros macMinBE e macMaxBE para o valor 1 .

A definição dos valores de $\operatorname{macMinBE}$ e macMaxBE adotados neste trabalho para os nós coordenadores foi baseada em experimentações por simulação. É importante salientar que, a definição correta dos valores de macMinBE e macMaxBE, a fim de priorizar o acesso ao meio de nós coordenadores, depende da configuração de formação de rede utilizada (por exemplo, número máximo de $\mathrm{CHs}$ filhos e número máximo de nós filhos associados).

\section{Avaliação de Desempenho}

Esta Seção apresenta uma avaliação por simulação do mecanismo de disseminação do tráfego de controle proposto neste artigo. O principal objetivo é analisar o comportamento do tráfego de controle quando aplicado os esquemas propostos, comparado com abordagens da Literatura que não os utilizam. Para esta avaliação de desempenho, foi utilizado o CT-SIM [21], um conjunto de modelos de simulação que implementa as principais funcionalidades de redes cluster-tree. CT-SIM é baseado no Castalia [22], um simulador de eventos discretos open-source para RSSFs e redes embarcadas gerais, desenvolvido pela National ICT Australia (NICTA) e baseado na plataforma OMNeT++.

\section{A. Ambiente de Simulação}

O cenário de simulação considera um ambiente com tamanho de $200 \mathrm{~m}$ x $200 \mathrm{~m}$, variando os cenários de acordo com o número de nós sensores: 101, 151, 201 e 251 nós sensores (sendo 1 coordenador PAN). Para todos os cenários, o coordenador PAN está localizado na posição central, enquanto que os outros nós sensores foram aleatoriamente distribuídos pelo ambiente.

A rede cluster-tree considera o esquema SDA (Superframe Duration Allocation) [17] para atribuir valores de Duração de Superframe (SD) e Intervalo de Beacons (BI), onde os valores para SDs são proporcionalmente alocados para cada $\mathrm{CH}$ de acordo com o número de nós descendentes. Com relação ao processo de formação da rede cluster-tree, cada $\mathrm{CH}$ pode associar no máximo 6 nós filhos, o que implica que, à medida que o número de nós aumenta (diferentes cenários), a profundidade e o número de clusters também aumentam.

Com relação ao tráfego de monitoramento (tráfego upstream), cada nó sensor (excluindo o coordenador PAN) gera 1.000 mensagens de dados, a uma taxa de 1 pacote a cada 20 segundos, que são enviados em direção ao coordenador PAN (nó sink da rede).

Com relação ao tráfego de controle (tráfego downstream), o coordenador PAN gera 100 mensagens de controle, a uma taxa de 1 pacote a cada 5 segundos, enviados para todos os $\mathrm{CHs}$ ao 
Tabela I

CONFiguraÇ̃̃o DA SimulaÇão.

\begin{tabular}{|c|c|}
\hline Descrição & Valor \\
\hline Área do ambiente & $200 \mathrm{~m} \times 200 \mathrm{~m}$ \\
\hline Número de nós sensores & $101 / 151 / 201 / 251$ \\
\hline Mensagens de Monitoramento (por nó) & 1.000 pacotes \\
\hline Taxa de Mensagens de Monitoramento & 1 pacote a cada $20 \mathrm{~s}$ \\
\hline Mensagens de Controle (coordenador PAN) & 100 pacotes \\
\hline Taxa de Mensagens de Controle & 1 pacote a cada $5 \mathrm{~s}$ \\
\hline Modelo de Rádio & Chipcon CC2420 \\
\hline Número de simulações (por cenário) & 20 execuções \\
\hline Intervalo de Confiança dos Resultados & $95 \%$ \\
\hline
\end{tabular}

longo da rede cluster-tree. É importante destacar que o tráfego de controle foi programado para ser gerado no momento da definição da janela de oportunidade Top-Down JTD. Para este estudo de simulação, a janela de oportunidade foi programada para iniciar após $1 \mathrm{~h}$ do início da simulação. Por uma questão de simplificação, apenas uma janela JTD foi definida para cada cenário de simulação. Tabela I sumariza as principais configurações da simulação.

O comportamento do mecanismo de escalonamento híbrido com mudança dos parâmetros CSMA-CA proposto neste artigo é comparado com diferentes esquemas de escalonamento utilizados na literatura. Por uma questão de conveniência, os seguintes acrônimos foram utilizados na apresentação dos resultados de simulação:

- Bottom-Up: abordagem que utiliza um esquema de escalonamento de rede puramente bottom-up, mantendo assim até o final da simulação.

- Top-Down: abordagem que utiliza um esquema de escalonamento de rede puramente top-down, mantendo assim até o final da simulação.

- Híbrido: abordagem que utiliza um esquema de escalonamento inicial bottom-up, alterando para o escalonamento top-down durante a janela de oportunidade. Entretanto, nessa abordagem, não é utilizado o esquema de alteração dos parâmetros CSMA-CA para a transmissão de mensagens de controle. Com isso, será avaliado o impacto de alterar os valores dos parâmetros CSMA-CA para o tráfego de controle.

- Híbrido + CSMA-CA: mecanismo proposto neste artigo, que considera um esquema de escalonamento inicial bottom-up, alterando para o escalonamento top-down, com mudança dos parâmetros CSMA-CA, durante a janela de oportunidade.

Para cada cenário de simulação e cada abordagem de escalonamento, as seguintes métricas de desempenho foram analisadas: atrasos médios de comunicação fim-a-fim e a taxa média de entrega de mensagens. Além disso, os resultados de simulação apresentados neste artigo são as médias de 20 diferentes execuções (com diferentes conjuntos de variáveis aleatórias), considerando um intervalo de confiança de $95 \%$.

\section{B. Resultados e Discussão}

Primeiramente, este estudo de simulação avaliou o impacto dos mecanismos propostos neste artigo no atraso médio de comunicação fim-a-fim das mensagens de controle, considerando os diferentes cenários de simulação. Figura 4 mostra os atrasos médios de comunicação fim-a-fim para o tráfego de controle, considerando as diferentes abordagens avaliadas.

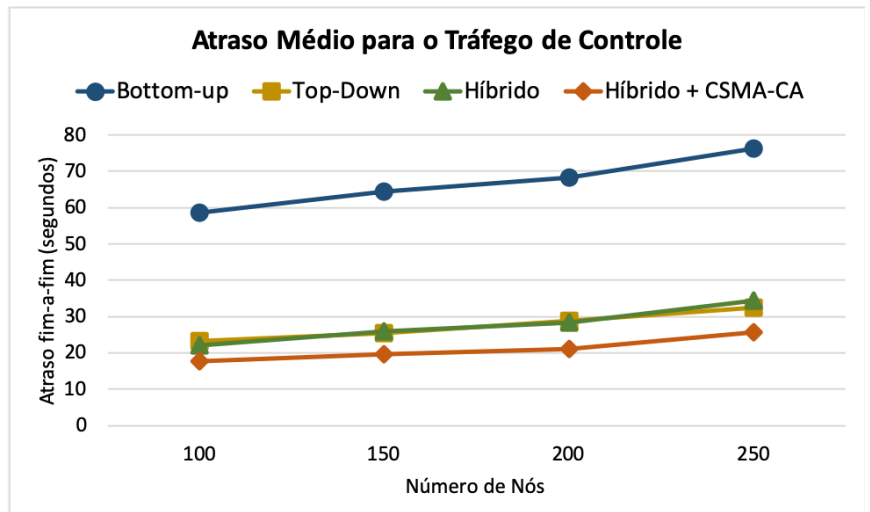

Figura 4. Atraso médio de comunicação fim-a-fim para as mensagens de controle, considerando as diferentes abordagens avaliadas.

Como pode ser observado, considerando que o mecanismo Híbrido + CSMA-CA proposto neste artigo cria janelas de oportunidade Top-Down e ajusta os parâmetros CSMA-CA a fim de priorizar o tráfego de controle, os atrasos de comunicação fim-a-fim para as mensagens de controle são notavelmente menores para todos os cenários, quando comparados com as outras abordagens avaliadas. Isso se deve ao fato de que a organização dos clusters seguindo um modelo top-down tende a priorizar o tráfego downstream, além de que uma configuração otimizada dos parâmetros CSMA-CA aumenta a probabilidade de acesso ao canal sem fio dos nós coordenadores, favorecendo assim uma rápida disseminação do tráfego de controle.

Note também que, como as abordagens Híbrido e Top-Down utilizam um esquema de escalonamento top-down, os atrasos médios para as mensagens de controle também são menores. Por outro lado, a abordagem Bottom-Up utiliza um esquema de escalonamento que prioriza o tráfego de monitoramento (tráfego upstream). Dessa forma, os atrasos médios enfrentados pelas mensagens de controle são considerados altos, pois precisam atravessar vários intervalos de beacons através do escalonamento de rede, o que mostra que esse esquema de escalonamento não é adequado para a disseminação de mensagens de controle.

Por sua vez, Figura 5 mostra a taxa de entrega média para o tráfego de controle, considerando as diferentes abordagens. De forma similar, o mecanismo Híbrido + CSMA-CA entrega praticamente todas as mensagens de controle (em média, acima dos $96 \%$ de taxa de entrega), nos diferentes cenários definidos, o que mostra a importância da modificação dos parâmetros CSMA-CA para garantir que os $\mathrm{CHs}$ possam disseminar com eficiência as mensagens de controle. Note que as outras abordagens possuem taxas de entregas menores do que a abordagem proposta neste artigo (em média, $8 \%$ menores), devido aos problemas de entrega de pacotes gerados pelo algoritmo de acesso com contenção ao canal sem fio. 


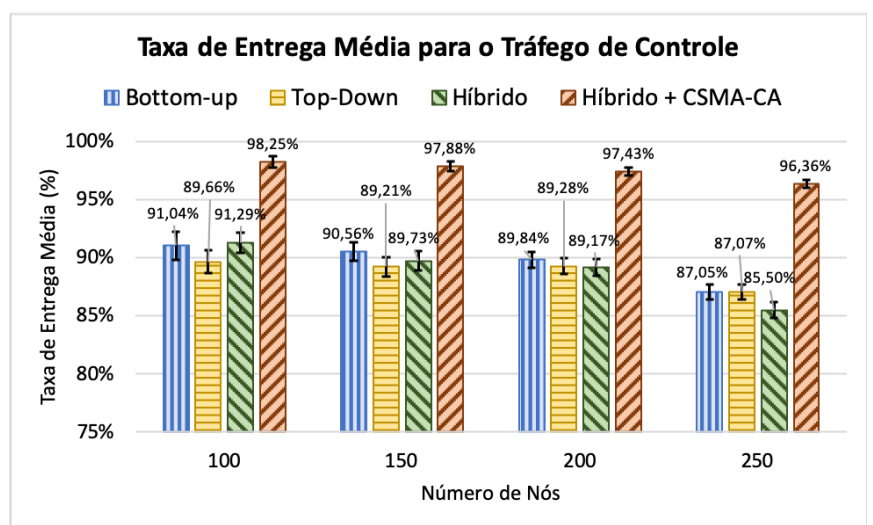

Figura 5. Taxa de entrega média para as mensagens de controle, considerando as diferentes abordagens avaliadas.

Além disso, também foi avaliado o comportamento de entrega de mensagens de controle por nível de profundidade na rede cluster-tree. Figura 6 mostra a entrega média de mensagens de controle para os $\mathrm{CHs}$ em diferentes profundidades. Por uma questão de espaço, a Figura 6 apresenta apenas a entrega média para as 3 primeiras profundidades (P1, P2 e P3) da rede cluster-tree.

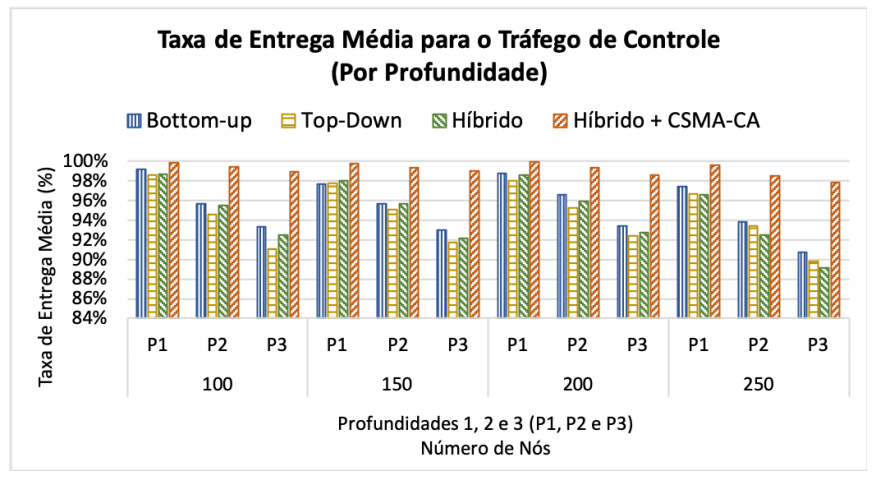

Figura 6. Taxa de entrega média das mensagens de controle por nível de profundidade, considerando as diferentes abordagens avaliadas.

Como pode ser notado, à medida que a profundidade de rede vai aumentando, a taxa de entrega média das mensagens de controle das abordagens Top-Down, Bottom-Up e Híbrido vai diminuindo. Isso mostra que a perda de mensagens devido à contenção vai se acumulando ao longo das profundidades da rede cluster-tree. Por sua vez, como o mecanismo Híbrido + CSMA-CA procura aumentar a probabilidade de acesso ao meio dos nós coordenadores, a taxa de entrega média das mensagens de controle se mantém favorável (diminui levemente), à medida que a profundidade de rede aumenta.

Uma questão importante é também avaliar o impacto do tráfego de controle sobre o tráfego de monitoramento gerado pelos nós sensores em redes cluster-tree. Para este propósito, um conjunto de simulações foi realizado considerando apenas o tráfego de monitoramento, com o intuito de capturar o comportamento das mensagens de monitoramento (atrasos de comunicação e taxa de entrega) nos diferentes cenários de simulação definidos. Figura 7 mostra os atrasos médios de comunicação fim-a-fim para o tráfego de monitoramento, considerando as diferentes abordagens e o ambiente apenas com tráfego de monitoramento.

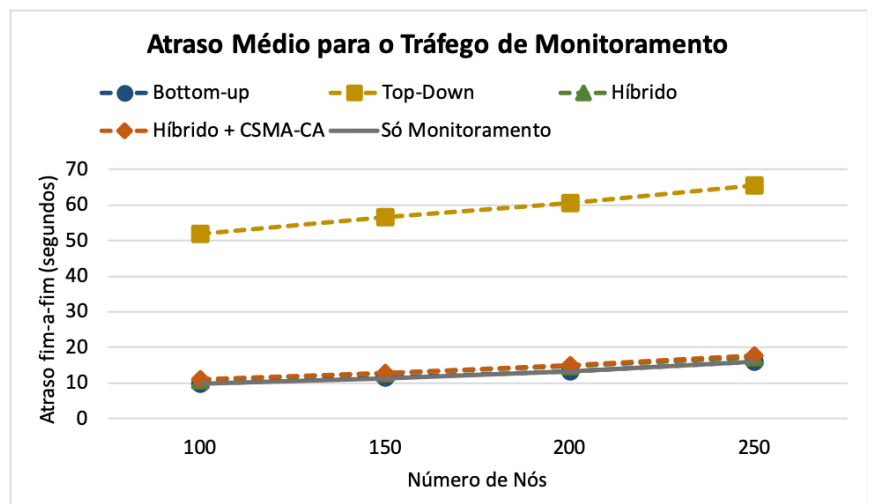

Figura 7. Atraso médio de comunicação fim-a-fim para as mensagens de monitoramento, considerando as diferentes abordagens avaliadas.

Como pode ser observado, apesar de acrescentar na rede um tráfego adicional (mensagens de controle), as abordagens Bottom-Up, Híbrido e Híbrido + CSMA-CA impactam minimamente nos atrasos médios de comunicação fim-a-fim do tráfego de monitoramento. Especificamente para a abordagem Híbrido + CSMA-CA, mesmo considerando o tráfego adicional de controle e priorizando o acesso ao meio para os nós coordenadores, com a intenção de priorizar o tráfego de controle, a abordagem proposta neste artigo mantém a qualidade de serviço para o fluxo de monitoramento (em média, um aumento de 1,5 segundos no atraso médio de comunicação), considerando todos os cenários de simulação. Por sua vez, a abordagem Top-Down impacta fortemente sobre o tráfego de monitoramento, pois o esquema de escalonamento topdown não prioriza o tráfego de upstream, aumentando assim de forma expressiva o atraso médio de comunicação para as mensagens de monitoramento.

Por fim, Figura 8 apresenta a taxa de entrega média para o tráfego de monitoramento, considerando as diferentes abordagens e o ambiente apenas com o tráfego de monitoramento. Note que todas as abordagens avaliadas forneceram qualidade na entrega das mensagens de monitoramento, mesmo adicionando um novo fluxo de mensagens (tráfego de controle), quando comparados ao ambiente de simulação que considera apenas o tráfego de monitoramento.

Portanto, os resultados de simulação apresentados mostram que o mecanismo Híbrido + CSMA-CA proposto neste artigo fornece Qualidade de Serviço para ambos os tráfegos de monitoramento e controle em redes cluster-tree, sendo assim adequados para típicas aplicações de monitoramento que necessitam de atividades periódicas de controle/atuação.

\section{CONCLUSÃO}

Este artigo apresenta um mecanismo eficiente para a rápida disseminação do tráfego de controle em RSSFs clustertree, sem impactar de forma relevante no típico tráfego de 


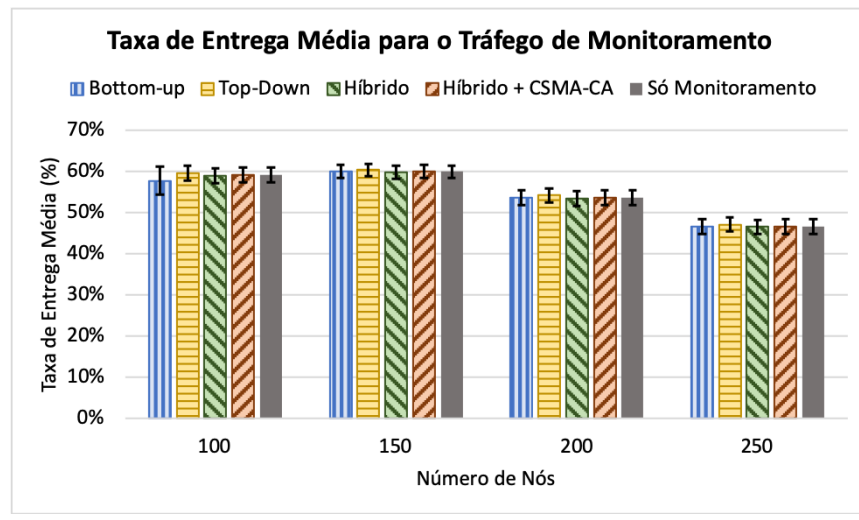

Figura 8. Taxa de entrega méida para as mensagens de monitoramento, considerando as diferentes abordagens avaliadas.

monitoramento gerado pelos nós sensores. A ideia básica é combinar um esquema de escalonamento híbrido de clusters, que define janelas de oportunidade Top-Down para priorizar o fluxo de mensagens downstream (tráfego de controle), com um esquema de configuração eficiente dos parâmetros CSMACA para os nós coordenadores, que aumenta a probabilidade de acesso ao canal sem fio desses dispositivos, favorecendo uma diminuição do tempo de disseminação e o aumento da taxa de sucesso de entrega de mensagens de controle.

Resultados de simulação mostram que a definição de janelas de oportunidade Top-Down para disseminação do tráfego de controle, combinado com uma configuração eficiente dos valores dos parâmetros do algoritmo CSMA-CA para os nós coordenadores, especificamente macMinBE e macMaxBE, podem reduzir expressivamente os atrasos de comunicação fim-a-fim e aumentar a taxa de entrega para mensagens de controle. Além disso, os resultados mostram que, mesmo adicionando um novo fluxo de dados à rede cluster-tree, o mecanismo proposto não impacta de forma relevante no típico tráfego de monitoramento, mesmo em ambientes com uma maior densidade de nós sensores. Assim, o mecanismo proposto neste artigo torna-se uma opção atraente para RSSFs de monitoramento, que necessitam de atividades de controle/atuação períodicas.

Como trabalhos futuros, o mecanismo proposto neste artigo será estendido para uma abordagem orientada a eventos para atividades de controle em RSSFs cluster-tree. Com isso, o fluxo de controle só seria configurado de acordo com a necessidade de atuação imposta pelas aplicações.

\section{ACKNOWLEDGMENT}

Os autores gostariam de agradecer o apoio financeiro da FAPEPI/MCT/CNPq/CT-INFRA (No 007/2018 - Programa PPP), do Conselho Nacional de Desenvolvimento Científico e Tecnológico CNPq (projeto 443711/2018-6) e da Universidade Federal do Piauí - UFPI (PIBIC - PICCN4910-2018).

\section{REFERÊNCIAS}

[1] J. A. Stankovic, A. D. Wood, and T. He, "Realistic Applications for Wireless Sensor Networks." Theoretical Aspects of Distributed Computing in Sensor Networks, no. 25, pp. 835-863, 2011.
[2] M.-H. Afsar, M. Mehdi e Tayarani-N, "Clustering in sensor networks," Journal of Network and Computer Applications, vol. 46, no. C, pp. 198226, Nov. 2014

[3] A. A. S. Kumar, K. Ovsthus, and L. M. Kristensen, "An Industrial Perspective on Wireless Sensor Networks - A Survey of Requirements, Protocols, and Challenges," IEEE Communications Surveys and Tutorials, vol. 16, no. 3, pp. 1391-1412, 2014.

[4] M. H. Rashid, Bushra e Rehmani, "Applications of wireless sensor networks for urban areas: A survey," Journal of Network and Computer Applications, vol. 60, no. C, pp. 192-219, Jan. 2016.

[5] IEEE 802.15.4, "IEEE Standard for Low-Rate Wireless Personal Area Networks (WPANs)," IEEE Computer Society, pp. 1-709, Dec. 2015.

[6] ZigBee, "ZigBee Specification," ZigBee Alliance (Document 053474r20), Sep. 2012.

[7] C. Li, H. Zhang, B. Hao, and J. Li, "A Survey on Routing Protocols for Large-Scale Wireless Sensor Networks," Sensors, vol. 11, no. 12, pp. 3498-3526, Dec. 2011.

[8] H. Lin, L. Wang, and R. Kong, "Energy Efficient Clustering Protocol for Large-Scale Sensor Networks," IEEE Sensors Journal, vol. 15, no. 12, pp. 7150-7160, 2015.

[9] M.-S. Yeh, Lun-Wu e Pan, "Beacon Scheduling for Broadcast and Convergecast in ZigBee Wireless Sensor Networks," Computer Communications, vol. 38, pp. 1-12, Feb. 2014.

[10] A. Koubaa, M. Alves, B. Nefzi, and Y.-Q. Song, "Improving the IEEE 802.15.4 Slotted CSMA/CA MAC for Time-Critical Events in Wireless Sensor Networks," in Proceedings of the Workshop of Real-Time Networks (RTN 2006), Satellite Workshop to ECRTS 2006, Dresden, Germany, Jul. 2006.

[11] L. Constante, J. Lau, R. Moraes, G. Araujo, C. Montez, and E. Leão, "Enhanced association mechanism for ieee 802.15.4 networks," in 2017 22nd IEEE International Conference on Emerging Technologies and Factory Automation (ETFA), Sep. 2017, pp. 1-8.

[12] A. Koubaa, A. Cunha, M. Alves, and E. Tovar, "TDBS: A Time Division Beacon Scheduling Mechanism for ZigBee Cluster-Tree Wireless Sensor Networks," Real-Time Systems, vol. 40, no. 3, pp. 321-354, Dec. 2008.

[13] P. Hanzalek, Z e Jurcík, "Energy Efficient Scheduling for Cluster-Tree Wireless Sensor Networks With Time-Bounded Data Flows: Application to IEEE 802.15.4/ZigBee," IEEE Transactions on Industrial Informatics, vol. 6, no. 3, pp. 438-450, 2010.

[14] R. Severino, N. Pereira, and E. Tovar, "Dynamic Cluster Scheduling for Cluster-tree WSNs," SpringerPlus, vol. 3, no. 1, p. 493, Aug. 2014.

[15] A. Koubaa, M. Alves, and M. Attia, "Collision-free beacon scheduling mechanisms for IEEE 802.15. 4/Zigbee cluster-tree wireless sensor networks," Technical Report (TR-061104), 2006.

[16] F. Xia, J. Li, R. Hao, X. Kong, and R. Gao, "Service Differentiated and Adaptive CSMA/CA over IEEE 802.15.4 for Cyber-Physical Systems," Scientific World Journal, vol. 2013, pp. 1-12, 2013.

[17] E. Leão, C. Montez, R. Moraes, P. Portugal, and F. Vasques, "Superframe Duration Allocation Schemes to Improve the Throughput of Cluster-Tree Wireless Sensor Networks," Sensors, vol. 17, no. 2, p. 249, Feb. 2017.

[18] E. Leão, V. Vasconcelos, P. Portugal, C. Montez, and R. Moraes, "A hybrid beacon scheduling scheme to allow the periodic reconfiguration of large-scale cluster-tree wsns," in 2018 IEEE 16th International Conference on Industrial Informatics (INDIN), July 2018, pp. 169-174.

[19] C. Benzaïd, M. Bagaa, and M. Younis, "Efficient clock synchronization for clustered wireless sensor networks," Ad Hoc Networks, vol. 56, pp. $13-27,2017$.

[20] A. Ghaffari, "Congestion control mechanisms in wireless sensor networks: A survey," Journal of Network and Computer Applications, vol. 52, pp. $101-115,2015$.

[21] E. Leão, R. Moraes, C. Montez, P. Portugal, and F. Vasques, "CTSIM: A simulation model for wide-scale cluster-tree networks based on the IEEE 802.15.4 and zigbee standards," International Journal of Distributed Sensor Networks, vol. 13, no. 3, pp. 1-17, 2017.

[22] Y. Tselishchev, A. Boulis, and L. Libman, "Experiences and lessons from implementing a wireless sensor network mac protocol in the castalia simulator," in 2010 IEEE Wireless Communication and Networking Conference, April 2010, pp. 1-6. 